\title{
How to Measure Gravitational Aberration?
}

\author{
Michal Křížek ${ }^{1}$ and Alena Šolcová ${ }^{2}$ \\ ${ }^{1}$ Mathematical Institute, Academy of Sciences,Žitná 25, CZ-115 67 Prague 1, Czech Republic \\ e-mail: krizek@mbox.cesnet.cz \\ ${ }^{2}$ Department of Mathematics, Faculty of Civil Engineering, Czech Technical University, \\ Thákurova 7, CZ-166 29 Prague 6, Czech Republic \\ e-mail: solcova@mbox.cesnet.cz
}

\begin{abstract}
In 1905, Henri Poincaré predicted the existence of gravitational waves and assumed their speed equal to the speed of light. If additionally the gravitational aberration would have the same magnitude as the aberration of light, we would observe several paradoxical phenomena. For instance, the orbit of two bodies would be unstable, since two attractive forces arise that are not in line and hence form a couple. This will be modelled by a nonautonomous system of ordinary differential equations with delay. In fact, any positive value of the gravitational aberration increases the angular momentum of such a system and this may contribute to the expansion of the universe. We found a remarkable coincidence between the Hubble constant and the increasing distance of the Moon from the Earth.

In 2000, Carlip showed that in general relativity gravitational aberration is almost cancelled out by velocity-dependent interactions. We show how the actual value of the gravitational aberration can be obtained by measurement of a single angle at a suitable time $t^{*}$ corresponding to the perihelion of an elliptic orbit. We also derive an a priori error estimate that expresses how accurately $t^{*}$ has to be determined to obtain the gravitational aberration to a prescribed tolerance.
\end{abstract}

Keywords. gravitation, causality, close binaries, Moon, Hubble constant, numerical methods

\section{Historical remarks}

Aberration of light is an apparent change of the observed position of some celestial body due to the movement of an observer, and the finite speed of light. The annual light aberration (Ron \& Vondrák 1986) caused by the Earth's orbit around the Sun was discovered by James Bradley around 1725 and published in Phil. Trans. (Bradley 1729). Throughout the year each star (except for the Sun) on the celestial sphere circumscribes an apparent ellipse whose semimajor axis in angular measure is $\tilde{\alpha} \doteq 10^{-4} \doteq 20^{\prime \prime}$. This phenomenon helped to improve the calculation of the speed of light to $c=3 \cdot 10^{5} \mathrm{~km} / \mathrm{s}$ from the relation

$$
c=\frac{v}{\tan \alpha} \doteq \frac{v}{\alpha} \quad,
$$

where $v \doteq 30 \mathrm{~km} / \mathrm{s}$ is the mean speed of the Earth and the light aberration angle $\alpha$ is given in radians. For a circular orbit of the Earth we would get $\tilde{\alpha}=\alpha$ (cf. also (5.9)). In Section 3, we introduce a formula similar to (1.1), where the gravitational aberration appears.

Gravitational waves were predicted already by Henri Poincaré. In 1905 he conjectured that their speed is the same as the speed of the light (see Poincaré 1905, p. 1507), i.e., before the same result was postulated by A. Einstein. If these speeds differ then it will be difficult to identify the source of gravitational waves with its optical counterpart, e.g., during (unsymmetric) explosions of supernovae. At present, several large projects (GEO, LIGO, VIRGO, LISA,...) are developed to measure the speed of gravitational waves and determine the direction from where they come. However, for the time being they have not been detected, even though all close binary systems are supposed to produce these waves. 


\section{Stability of a close binary system}

By analyzing the motion of the Moon, Pierre Laplace conjectured in 1805 (see Laplace 1966) that the actual speed of Newtonian gravity $c_{g}$ must be at least $7 \times 10^{6} \mathrm{c}$. Recently, van Flandern (1998) raised this lower bound to $2 \times 10^{10} c$; otherwise two bodies forming a binary system could not have stable orbits. $\dagger$ For $c_{g} \approx c$ their angular momenta would not be in equilibrium.

If the Sun attracts Jupiter at its instantaneous position and also Jupiter attracts the Sun at its instantaneous position (i.e., $c_{g}=\infty$ ), then these forces are in the same line and in balance. Note that the centre of gravity of this system lies outside the Sun.

On the other hand, suppose that the Sun $S$ attracts Jupiter towards its previous position $J^{\prime}$ (see Figure 1). Similarly let Jupiter $J$ attract the Sun in the direction of its previous position $S^{\prime}$ (i.e., $c_{g}<\infty$ ). Then a couple of forces arises which increases the angular momentum and total energy of this system (see Křížek 1999), and thus also prolongs the orbital period, which does not fit with observations. Therefore, the Sun's rays arriving at the Earth are not parallel with the vector of attractive gravitational force of the Sun. Ways of interpreting the above paradoxical phenomena that do not contradict general relativity and causality are discussed in Carlip (2000), Marsh \& Nissim-Sabat (1999), and van Flandern (1998).

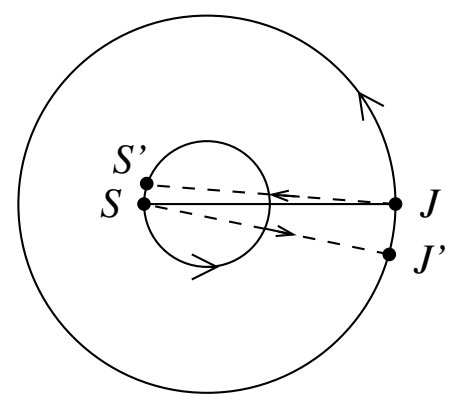

Figure 1. Gravitational interaction between the Sun and Jupiter for $c_{g}<\infty$.

Now let us verify the Laplace conjecture numerically. Consider two mass points $m_{1}$ and $m_{2}$ in the two- or three-dimensional space equipped with the Euclidean norm $|\cdot|$. Introducing a delay into gravitational interactions, the classical autonomous Newtonian system of ordinary differential equations can be rewritten as the following nonautonomous system for two trajectories $r_{1}$ and $r_{2}$ :

$$
\begin{aligned}
& r_{1}^{\prime \prime}(t)=G \frac{m_{2}\left(r_{2}\left(t-d_{2}(t)\right)-r_{1}(t)\right)}{\left|r_{2}\left(t-d_{2}(t)\right)-r_{1}(t)\right|^{3}}, \\
& r_{2}^{\prime \prime}(t)=G \frac{m_{1}\left(r_{1}\left(t-d_{1}(t)\right)-r_{2}(t)\right)}{\left|r_{1}\left(t-d_{1}(t)\right)-r_{2}(t)\right|^{3}}
\end{aligned}
$$

with two variable delays $d_{1}$ and $d_{2}$ satisfying (2.4), and the gravitational constant $G=6.673$. $10^{-11} \mathrm{~m}^{3} \mathrm{~kg}^{-1} \mathrm{~s}^{-2}$. Consider the initial conditions:

$$
r_{i}(t)=p_{i}(t), \quad r_{i}^{\prime}(t)=v_{i}(t), \quad t \in[\tau, 0], \quad i=1,2,
$$

where $\tau \leqslant 0$ is an appropriate given number and $p_{i}$ and $v_{i}$ are given functions characterizing previous positions and velocities. If $c_{g}=\infty$ then $\tau=d_{1}=d_{2}=0$ and system (2.1)-(2.2) reduces to the classical Newton two-body problem. For $c_{g}<\infty$ the delay functions satisfy the relations

$$
d_{1}(t)=\frac{\left|r_{1}\left(t-d_{1}(t)\right)-r_{2}(t)\right|}{c_{g}}, \quad d_{2}(t)=\frac{\left|r_{2}\left(t-d_{2}(t)\right)-r_{1}(t)\right|}{c_{g}},
$$

$\dagger$ Note that not all interactions have the same speed. For instance the speed of the weak interaction is much smaller than that of light, since the masses of the gauge bosons $W^{ \pm}$and $Z^{0}$ are more than 80 times larger than the proton mass. 
i.e., each $d_{i}$ has to be calculated iteratively in the numerical solution (using the Banach fixedpoint theorem). For the theory of ordinary delay differential equations we refer to Driver (1977), Louisell (1991), and Meinardus \& Nürnberger (1985).

Assume now that $m_{1} R_{1}=m_{2} R_{2}$, where $R_{1}$ and $R_{2}$ are distances from the Newtonian centre of gravity. Let us define

$$
p_{1}=\left(R_{1}, 0\right), p_{2}=\left(-R_{2}, 0\right), v_{1}=\left(0, \frac{\sqrt{G m_{2} R_{1}}}{R_{1}+R_{2}}\right), v_{2}=\left(0,-\frac{\sqrt{G m_{1} R_{2}}}{R_{1}+R_{2}}\right) .
$$

These values yield exactly circular orbits for $\tau=0$ in (2.3) and $c_{g}=\infty$. They are employed to establish initial conditions (2.3) for $c_{g}<\infty$. This, of course, requires to store old values of $r_{1}$ and $r_{2}$ throughout the computation due to the initial conditions (2.3). A big advantage of computer simulation is that we can easily perform many tests for various possible values of the speed of gravity $c_{g}$ appearing in (2.4).

For $m_{1}=m_{2}$ and $c_{g} \ll c$ the numerically calculated trajectories $r_{1}$ and $r_{2}$ depicted in Figure 2 are quite unrealistic, since they form two quickly expanding spirals.

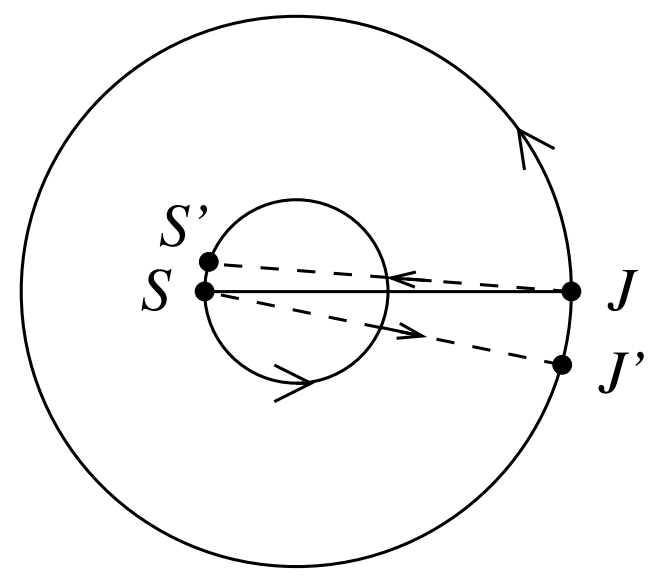

Figure 2. Trajectories of a two-body problem (2.1)-(2.4) for $m_{1}=m_{2}$ and $c_{g} \ll c$.

As another illustrative example consider the close binary system Earth-Moon with

$$
m_{1}=5.976 \cdot 10^{24} \mathrm{~kg}, \quad m_{2}=7.350 \cdot 10^{22} \mathrm{~kg},
$$

and the corresponding distance

$$
D=R_{1}+R_{2}=384400 \mathrm{~km} .
$$

For $c_{g}=c$ we calculate that the distance between Moon and Earth will be about $250 \mathrm{~m}$ larger after 1 year (i.e., after 12 revolutions), which does not correspond with observations. If for instance $c_{g}=10^{5} \mathrm{c}$, this augmentation is only

$$
a=2.5 \mathrm{~cm} / \mathrm{yr}
$$

and the associated trajectories form two very slowly expanding spirals. $\dagger$

By means of laser retroreflectors installed on the Moon by Apollo 11, Lunokhod, Apollo 14, etc., we know that the mean distance between Earth and Moon increases by $3.8 \mathrm{~cm}$ per year. However, this value depends not only on the finite speed of gravity, but also on tidal forces (see Section 5 for details) and other phenomena that have an influence on the variable distance and

$\dagger$ Similar phenomena are observed when the standard gravitational potential $1 / r$ is replaced by $1 / r \pm C / r^{2}$. In this case trajectories again expand and moreover, a perihelion advance of elliptic orbits appears (see Krř́žek 1999). 
that are not described by (2.1)-(2.4). Finite speed of gravity thus always causes an expansion of any binary or multiple body system and it should be taken into account when dealing with the expansion of the whole universe (see Krrížek 1999). A high value of $c_{g}$ could also explain a perfect point-symmetry of many galaxies.

All calculations were done in extended 10-byte precision by the standard fourth order explicit Runge-Kutta method (see Ralston \& Rabinowitz 2001) which gives a surprisingly small discretization error when the orbits are circular (Křížek 1995, Kř́žek 1999). For instance, the time step $\Delta t=100 \mathrm{~s}$, that produces practically the same results as $\Delta t / 2$, yields for $c_{g}=\infty$ a total error after 1000 revolutions of only $17 \mathrm{~mm}$, which is almost negligible.

\section{Gravitational aberration}

In this section we introduce the concept of gravitational aberration. We first restrict ourselves to measurement on the Earth. For simplicity assume now that its orbit is elliptic. By Kepler's second law the radius vector connecting the Earth and the Sun sweeps out equal areas in equal times, and therefore, $\frac{1}{2} r v T=\pi a b$, where $T=31558149.5 \mathrm{~s}(=365.25636$ days $)$ is the Earth's sidereal year,

$$
a=149.598 \cdot 10^{6} \mathrm{~km}, \quad b=149.577 \cdot 10^{6} \mathrm{~km}
$$

is the semimajor and semiminor axis, respectively, $r=147.055 \cdot 10^{6} \mathrm{~km}$ is the distance of the Earth to the Sun at the perihelion $P$ of its elliptic orbit and $v$ is the magnitude of the velocity at $P$, i.e.,

$$
v=\frac{2 \pi a b}{r T}=30.287 \mathrm{~km} / \mathrm{s} .
$$

The associated value of the light aberration $\alpha$ at $P$ is then given by (cf. (1.1))

$$
\alpha=\frac{v}{c}=0.000101027=20^{\prime \prime} .84 \text {. }
$$

Further assume that the vector of gravitational interaction is perpendicular to the Earth's trajectory at perihelion $P$. Denote by $\beta$ the angle between the Sun's rays arriving at the Earth and the tangent vector to the Earth's trajectory when the Earth is at $P$ (around January 4 ). The tangent vector lies on a line connecting two opposite points on the ecliptic. The gravitational aberration $\gamma$ at $P$ is then given by the formula

$$
\gamma=\alpha+\beta-\frac{\pi}{2}
$$

where $\alpha$ is defined by (3.2) and $\beta$ has to be measured (cf. Figure 3).

Similarly to (1.1) we can define the real "Newtonian" speed of gravity

$$
c_{g}=\frac{v}{\tan \gamma} \doteq \frac{v}{\gamma} .
$$

For $\gamma=0$ the speed of gravitational interaction would be infinite. However, the actual value of $\gamma$ must be at least slightly positive, if we want to preserve causality. For instance, the angles $\angle J S J^{\prime}$ and $\angle S J S^{\prime}$ representing the gravitational aberration in Figure 1 cannot vanish when $c_{g}<\infty$. From (1.1) and (3.4) we obtain the formula

$$
c_{g}=\frac{\alpha c}{\gamma} .
$$

Note that ?2) showed that in general relativity gravitational aberration is almost cancelled out by velocity-dependent interactions. Therefore, we do not see the Sun in the direction of its attractive force, but slightly shifted.

\section{A priori estimation of reaching the perihelion}

The disadvantage of the method described in Section 3 is the fact that the Earth's trajectory is substantially influenced by the Moon. Therefore, it is much more suitable to measure the 


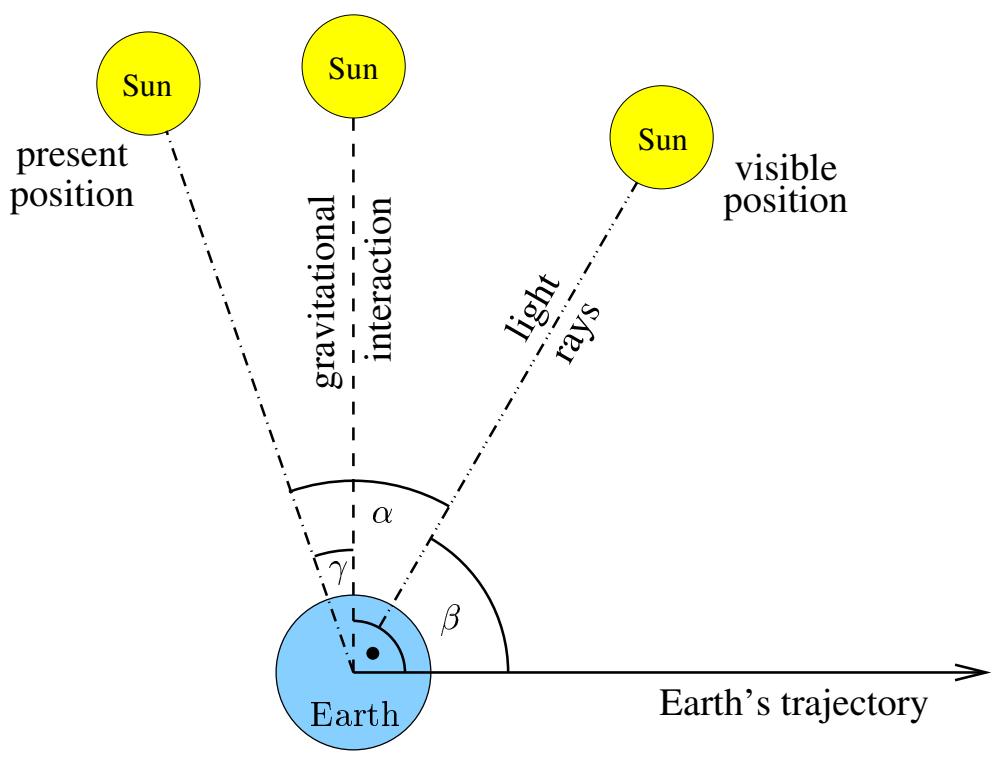

Figure 3. The speed of gravitational interaction $c_{g}$ can be determined from only the knowledge of the angle $\beta$, when the Earth is at the perihelion.

angle $\beta$ on a spacecraft orbiting the Sun. If its trajectory is circular, we can use relations (3.5) and (3.3) for any time.

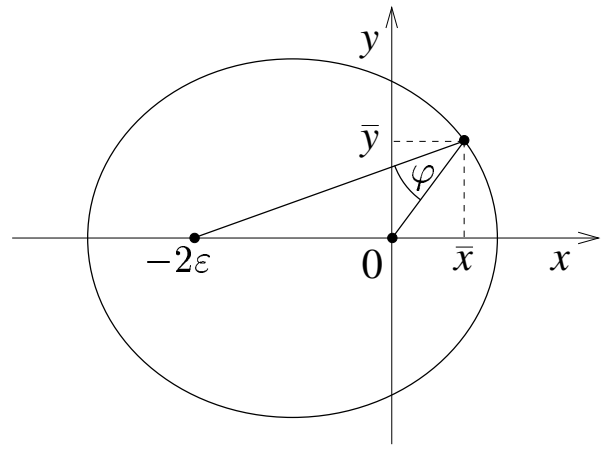

Figure 4. Coordinates of a spacecraft with an elliptic orbit.

Suppose now that the spacecraft trajectory is elliptic. Let us ask the question: how accurately do we have to measure the instant of reaching the perihelion to determine the gravitational aberration $\gamma$ with an error less than a given angle, e.g., $1^{\prime \prime}$. To answer this, let the Sun be placed at the origin of the coordinate system and let the second focus be at the point $(-2 \varepsilon, 0)$, where

$$
\varepsilon=\sqrt{a^{2}-b^{2}}
$$

and $a>b$. Denote by $(\bar{x}, \bar{y})$ the coordinates of the spacecraft (see Figure 4). Let $k_{1}$ and $k_{2}$ be the slopes of two lines passing through the foci $(0,0)$ and $(-2 \varepsilon, 0)$, respectively, and the point $(\bar{x}, \bar{y})$. Substituting $\bar{y}=k_{1} \bar{x}$ into the equation of the ellipse $b^{2}(\bar{x}-\varepsilon)^{2}+a^{2} \bar{y}^{2}=a^{2} b^{2}$, we get a quadratic equation for $\bar{x}>0$ :

$$
\left(a^{2} k_{1}^{2}+b^{2}\right) \bar{x}^{2}-2 b^{2} \varepsilon \bar{x}-a^{2} b^{2}=0 .
$$


The second slope can be then expressed as (see Figure 4)

$$
k_{2}=\frac{k_{1} \bar{x}}{\bar{x}+2 \varepsilon} .
$$

For the angle $\varphi$ between these two lines we have

$$
\tan \varphi=\left|\frac{k_{1}-k_{2}}{1+k_{1} k_{2}}\right|, \quad k_{1} k_{2} \neq-1,
$$

where by substituting for $k_{2}$ from relation (4.3) we obtain the expression for the angle $\varphi$ as a function of a single variable $k_{1}$. Thus we get:

Corollary. Under the above notations for elliptic orbit we have

$$
\varphi=\arctan \left|\frac{2 \varepsilon k_{1}}{\bar{x}+2 \varepsilon+k_{1}^{2} \bar{x}}\right|,
$$

where $\varepsilon$ is given by (4.1) and $\bar{x}>0$ satisfies equation (4.2).

Let us apply this Corollary to the elliptic orbit whose semi-axes are given by (3.1). According to Rektorys (1994), p. 116, a normal vector at the point $(\bar{x}, \bar{y})$ bisects the angle $\varphi$. From this we may calculate the difference $\frac{1}{2} \varphi$ between the slopes of the normal and $k_{1}$. Denote by $t^{*}$ the time instant when the spacecraft is at the perihelion. According to (4.4), $\frac{1}{2} \varphi$ will be less than $1^{\prime \prime}$ for $\left|k_{1}\right|<0.0006$. This slope corresponds to the time interval $\left(t^{*}-50, t^{*}+50\right)$ in minutes.

The spacecraft's orbit is influenced by other planets (except for the Earth). In particular, Jupiter affects the orbit by a force which is at most 16000 times smaller than that of the Sun. We are, of course, not able to take into account all nongravitational forces that have an influence on the measurement (even though almost negligible), such as the solar wind, thermal radiation of the Earth, Yarkovsky effect, collision with interplanetary dust and meteorites, presence of magnetic fields, gravitational radiation, etc. Anyway, an approximate value of $c_{g}$ can also be similarly obtained by (3.5) for the other planets, asteroids, comets etc. with the corresponding aberration $\gamma$. The closer approach of the spacecraft to the Sun, the larger gravitational aberration should appear.

\section{Some remarkable coincidences}

From Section 2 we know that an arbitrarily small value of the gravitational aberration of a binary system tends to increase its angular momentum. The associated trajectories form two very slowly expanding spirals. Similar phenomena are observed for a general $n$-body problem as well. Thus, the finite speed of gravitational interaction can contribute to the expansion of the universe which is given by the Hubble constant

$$
H_{0}=\frac{c}{15 \mathrm{Gly}}=20 \mathrm{~km} \mathrm{~s}^{-1}(\mathrm{Mly})^{-1},
$$

where 15 Gly is an approximate value of the age of universe 13.7 Gly.

For the mean distance $D$ of the Moon from the Earth given by (2.7), we can compare the Hubble constant with the increasing value of $D$ during one year $T=31558149.5 \mathrm{~s}=1 \mathrm{yr}$,

$$
\begin{gathered}
H_{0}=20 \mathrm{~km} \mathrm{~s}^{-1}(\mathrm{Mly})^{-1}=2 \mathrm{~cm} \mathrm{~s}^{-1} \mathrm{ly}^{-1}=2 T \mathrm{~cm} \mathrm{yr}^{-1} \mathrm{ly}^{-1} \\
=\frac{2 T}{9.46 \cdot 10^{12}} \mathrm{~cm} \mathrm{yr}^{-1} \mathrm{~km}^{-1}=\frac{2 T}{2.5 \cdot 10^{7} D} \mathrm{~cm} \mathrm{yr}^{-1}=\frac{2.5}{D} \mathrm{~cm} \mathrm{yr}^{-1} .
\end{gathered}
$$

We observe that this value is the same as that given in (2.8) and it is surprisingly very close to the measured value

$$
\Delta=3.8 \mathrm{~cm} / \mathrm{yr} \text {. }
$$

Now let us estimate the contribution of tidal forces to the value $\Delta$. Consider the system EarthMoon with masses $m_{1}$ and $m_{2}$ given in (2.6), $R_{1}=4670369.5 \mathrm{~m}$, and $R_{2}=379729630.5 \mathrm{~m}$ 
(see (2.7)). By conservation of the total momentum of this system, the value

$$
M=I_{1} \omega_{1}+I_{2} \omega_{2}+m_{1} R_{1} v_{1}+m_{2} R_{2} v_{2}
$$

has to be constant. Here $v_{1}=12.545 \mathrm{~m} / \mathrm{s}$ and $v_{2}=1020 \mathrm{~m} / \mathrm{s}$ is the speed of the Earth and Moon, respectively, relative to their centre of gravity,

$$
I_{1}=8.036 \pm 0.008 \cdot 10^{37} \mathrm{~kg} \mathrm{~m}^{2}
$$

is the inertia moment of the Earth (see Burša \& Pěč 1993), $\omega_{1}=2 \pi / T_{1}$ is the angular frequency of the Earth, and $T_{1}=86164 \mathrm{~s}$ is the sidereal day. Moreover, by Rektorys (1994), p. 109, we have

$$
I_{2} \omega_{2}<\frac{16}{15} \rho_{2} \frac{\pi^{2} r_{2}^{5}}{T_{2}}=0.236 \cdot 10^{30} \quad \mathrm{~kg} \mathrm{~m}^{2} \mathrm{~s}^{-1},
$$

where $\rho_{2}=3340 \mathrm{~kg} / \mathrm{m}^{3}$ denotes the mean density of the Moon, $r_{2}=1737 \mathrm{~km}$ is its radius, and $T_{2}=29.322 T_{1}$, i.e., the term $I_{2} \omega_{2}$ corresponding to the Moon is almost negligible when compared with $I_{1} \omega_{1}=0.586 \cdot 10^{34} \mathrm{~kg} \mathrm{~m}^{2} \mathrm{~s}^{-1}$.

The Earth's rotation slows down mainly due to tidal forces of the Moon $(\sim 66 \%)$, but also of the Sun $(\sim 33 \%)$ so that the length of a day increases by $\tau_{1}=1.7 \cdot 10^{-5}$ s per year (see e.g., Said \& Stephenson 1996, p. 270). Thus its angular frequency after one year will be

$$
\bar{\omega}_{1}=\frac{2 \pi}{T_{1}+\tau_{1}} .
$$

Therefore, by (5.4) and (5.6), the decrease of the Earth's angular momentum is

$$
I_{1}\left(\omega_{1}-\bar{\omega}_{1}\right)=2 \pi I_{1} \frac{\tau_{1}}{T_{1}\left(T_{1}+\tau_{1}\right)}=1.156 \cdot 10^{24} \quad \mathrm{~kg} \mathrm{~m}^{2} \mathrm{~s}^{-1} .
$$

The Moon also reduces its angular momentum due to (5.2) and the 1:1 resonance between the orbital period $T_{2}$ and the Moon's rotation. However, from (5.5) and Kepler's third law we find that the decrease of the Moon's angular momentum is negligible with respect to the value given in (5.7). Therefore, the decrease in (5.7) must be compensated by the increase of the sum $m_{1} R_{1} v_{1}+m_{2} R_{2} v_{2}$ in (5.3).

Since $m_{1} v_{1}=m_{2} v_{2}$, we get by (2.7) that

$$
m_{1} R_{1} v_{1}+m_{2} R_{2} v_{2}=\left(R_{1}+R_{2}\right) m_{1} v_{1}=D m_{1} v_{1} .
$$

Dividing $66 \%$ of the value given in (5.7) by $m_{1} v_{1}$, we find that the distance $D$ would increase only about

$$
\Delta_{1}=1 \mathrm{~cm} / \mathrm{yr}
$$

We observe that the difference

$$
\Delta_{2}=\Delta-\Delta_{1}=2.8 \mathrm{~cm} / \mathrm{yr}
$$

between the measured value $\Delta$ in (5.2) and the calculated value $\Delta_{1}$ corresponding to tidal forces is surprisingly very similar to the value $H_{0}$ given by (5.1). This enables us to declare that the gravitational aberration caused by the finite speed of gravity contributes not only to the increase of the distance Earth-Moon, but also to the expansion of the whole universe.

We close this section by discussing another interesting property. The direction of the Earth's movement changes every day with about $1^{\circ}\left(\doteq 360^{\circ} / 365.25\right.$ days $)$. Light photons travel from the Sun to the Earth in about 8.3 minutes. Consequently, during this time period the Earth shifts its trajectory about an angle

$$
\bar{\alpha} \doteq \frac{8.3}{60 \cdot 24 \cdot 365.25} 360^{\circ} \doteq 20^{\prime \prime}
$$

with respect to the stars. The angle $\bar{\alpha}$ is very small, and thus it is almost equal to its tangent (using radian measure). For a circular orbit we observe by (5.8) and (1.1) that the angles $\alpha$ and $\bar{\alpha}$ coincide:

$$
\bar{\alpha}=\frac{2 \pi r}{c T}=\frac{v}{c}=\alpha .
$$


This also means that we don't see the Sun at its instantaneous position, but about $\bar{\alpha} \doteq 20^{\prime \prime}$ delayed (see Figure 3). Note that the instantaneous position of the Sun is about $20^{\prime \prime}$ east of its visible position.

\section{Acknowledgements}

This paper was supported by Institutional Research Plan nr. AV0Z 10190503 and Grant nr. 1P05ME749 of the Ministry of Education of the Czech Republic. The authors are indebted to Jan Brandts (Amsterdam), Lawrence Somer (Washington, D.C.), Filip Kř́žek, Ctirad Matyska, Vojtěch Pravda, and Jan Vondrák from Prague for fruitful discussions.

\section{References}

Bradley, J. 1729, Phil. Trans 35, 637

Burša, M. \& Pěč, K. 1993, Gravity Field and Dynamics of the Earth, Springer, Berlin

Carlip, S. 2000, "Aberration and the speed of gravity", Phys. Lett. A, 267, 81-87

Driver, R.D. 1977, "Ordinary and delay differential equations", Appl. Math. Sci., vol. 20, Springer, Berlin

Kř́žzek, M. 1995, "Numerical experience with the three-body problem", J. Comput. Appl. Math., 63, 403-409

Křížek, M. 1999, "Numerical experience with the finite speed of Gravitational interaction", Math. Comput. Simulation, 50, 237-245

Laplace, P.S. 1966, A treatise in celestial mechanics, vol. IV, book X (translated by N. Bowditch), Chelsea, New York

Louisell, J. 1991, "A stability analysis for a class of differential-delay equations having timevarying delay", in: S. Busenberg, M. Marteli (eds.), Delay Differential Equations and Dynamical Systems, LN in Math., vol. 1475, Springer, Berlin, 225-242

Marsh, G.E. \& Nissim-Sabat, Ch. 1999, "Comment on 'The speed of gravity'", Phys. Lett. A, $262,257-260$

Meinardus, G. \& Nürnberger, G.N. (eds.) 1985, "Delay equations, approximation and applications", Internat. Ser. Numer. Math., vol. 74, Birkhäuser, Basel

Poincaré, H. 1905, "Sur la dynamique de l'électron", C. R. Acad. Sci. Paris, 140, 1504-1508

Ralston, A. \& Rabinowitz, P. 2001, A First Course in Numerical Analysis, Dover Publications, New York

Rektorys, K. 1994, Survey of Applicable Mathematics, vol. I, Kluwer, Dordrecht

Ron, C. \& Vondrák, J. 1986, "Expansion of annual aberration into trigonometric series", Bull. Astron. Inst. Czechosl., 37, 96-103

Said, S.S. \& Stephenson, F.R. 1996 "Solar and lunar eclipse measurements by medieval Muslim astronomers", J. Hist. Astronom., 27, 259-273

van Flandern, T. 1998, "The speed of gravity - what the experiments say", Phys. Lett. A, 250, $1-11$ 